\title{
Utility of color Doppler echocardiography combined with clinical markers in diagnosis and prediction of prognosis of coronary artery lesions in Kawasaki disease
}

\author{
MEI XUE and JING WANG \\ Department of Ultrasound Diagnosis, The Affiliated Hospital of \\ Inner Mongolia Medical University, Hohhot, Inner Mongolia Autonomous Region 010050, P.R. China
}

Received April 30, 2019; Accepted September 12, 2019

DOI: $10.3892 /$ etm.2020.8519

\begin{abstract}
The utility of color Doppler echocardiography in the diagnosis and follow-up of Kawasaki disease (KD) with coronary artery lesions (CAL) was analyzed, and the clinical parameters associated with the disease were examined. The general data, the color Doppler echocardiography data and the biochemical indexes from 102 children with KD were analyzed. The patients were divided into a CAL group and a non-coronary artery lesion (NCAL) group based on the presence or absence of CAL. The risk factors for CAL in KD were screened by univariate and multivariate analyses. Among the 102 cases, CAL complications were identified in 47 cases (46.08\%). Compared with the NCAL group, the CAL group showed significantly higher incidences of fever duration, increased levels of $\mathrm{N}$-terminal pro B-type natriuretic peptide (NT-proBNP), cardiac troponin I (cTnI), C-reactive protein (CRP), intravenous immunoglobulin resistance, erythrocyte sedimentation rate (ESR), platelets, alanine aminotransferase and aspartate aminotransferase, and significantly lower serum albumin levels $(\mathrm{P}<0.05)$. According to the multivariate analysis, fever duration [odds ratio $(\mathrm{OR})=2.014$ ], NT-proBNP (OR=3.004), cTnI level (OR=2.638), ESR (OR=1.461) and CRP elevation $(\mathrm{OR}=1.094)$ were predictors of $\mathrm{CAL}$ in KD. During convalescence, the left and right coronary artery diameters in the CAL group significantly decreased $(\mathrm{P}<0.05)$. Color Doppler echocardiography can observe the condition of
\end{abstract}

Correspondence to: Professor Jing Wang, Department of Ultrasound Diagnosis, The Affiliated Hospital of Inner Mongolia Medical University, 1 Tongdao North Street, Hohhot, Inner Mongolia Autonomous Region 010050, P.R. China

E-mail: wangjing49@126.com

Abbreviations: KD, Kawasaki disease; CAL, coronary artery lesion; NCAL, non-coronary artery lesion; NT-proBNP, N-terminal pro B-type natriuretic peptide; cTnI, cardiac troponin I; ESR, erythrocyte sedimentation rate; CRP, C-reactive protein; IVIG, intravenous immunoglobulin

Key words: KD, color Doppler Echocardiography, CAL, cTnI, CRP, NT-proBNP coronary artery disease in patients with KD in real time and predicts its outcomes, which may be helpful for early diagnosis and long-term follow-up. Fever duration, cTnI, NT-proBNP and ESR levels were correlated with coronary artery diameter, of which the comprehensive use may be more accurate in determining the occurrence of CAL in KD.

\section{Introduction}

Kawasaki disease (KD) is a self-limiting, acute disease characterized by fever, rash and vasculitis. In total, $\sim 85 \%$ of patients with KD are younger than 5 years old, with an average age of 2 years. The prevalence of KD among children under 5 years of age is $>50 / 100,000$ in areas, such as Korea, Japan and Taiwan. The prevalence has increased over the past 20 years for unknown reasons (1). If patients with $\mathrm{KD}$ are not correctly diagnosed on time and treated accordingly, it may result in the formation of coronary artery lesions (CAL). A Previous study demonstrated that the risk for untreated children with $\mathrm{KD}$ younger than 1 year developing CAL is $>60 \%$ (2).

As KD can only be diagnosed by identifying the clinical symptoms, the diagnosis and treatment of patients with KD are sometimes delayed, especially in patients with incomplete KD, which is characterized by incomplete manifestation of clinical symptoms. Therefore, in cases with vague clinical symptoms, diagnosis must rely on auxiliary diagnostic markers, such as increased C-reactive protein (CRP) levels and erythrocyte sedimentation rate (ESR) levels, and low albumin, anemia and alanine aminotransferase (ALT) levels $(1,2)$.

Previously, developments in molecular biology and laboratory medicine have promoted the understanding of the association between KD and clinical indicators, including $\mathrm{N}$-terminal pro-B-type natriuretic peptide (NT-proBNP) and cardiac troponin I (cTnI). Monitoring the dynamic variations of NT-proBNP, cTnI and other clinical indicators in patients with $\mathrm{KD}$ is effective for evaluating the risk of CAL and predicting prognosis (3). Color Doppler echocardiography is an imaging technique utilized to identify coronary artery anomalies and assess myocardial function. Coronary artery diameter and its Z-score are useful in diagnosis of patients with KD and CAL risk stratification (4). Children with KD, particularly infants, tend to have atypical signs and symptoms, which makes it diffi- 
cult to diagnose. Therefore, color Doppler echocardiography is particularly critical for diagnosis of KD in this population group (5). To the best of the authors' knowledge, only a few previous studies have evaluated the association of color Doppler ultrasonography and clinical indicators in patients with KD with CAL $(5,6)$. Therefore, the present study aimed to analyze the benefit of color Doppler echocardiography combined with clinical indicators in the diagnosis and prognosis prediction of KD complicated with CAL.

\section{Patients and methods}

Patients. The present study recruited 102 children with KD hospitalized in The Affiliated Hospital of Inner Mongolia Medical University from September 2012 to October 2018. This prospective work was approved by The Ethics Committee of the Affiliated Hospital of Inner Mongolia Medical University, and informed consents were obtained from the legal guardians of the enrolled children. KD was diagnosed according to the criteria recommended by The American Heart Association, $2004(7,8)$. Inclusion criteria consisted of patients who were: i) Younger than 18 years old; ii) signed informed consent; and iii) met the diagnostic criteria for KD in children. Exclusion criteria consisted of patients with: i) Insufficient clinical data; ii) inherited metabolic illnesses and developmental malformations; and iii) cardiopulmonary insufficiency or other critical illnesses. The Z-score of the coronary artery diameter was set with respect to the body surface area of patients in the CAL group, and it was set as $\geq 2.5$. However, it was set to $\leq 2.5$ in the non-coronary artery lesion (NCAL) group (9).

Clinical examination. Whole blood, plasma and serum samples were collected from patients with acute KD before any treatment with intravenous immunoglobulin (IVIG). White blood cell count, blood platelet count, IVIG resistance, NT-proBNP, hemoglobin, serum albumin (ALB), cTnI, CRP, ESR, ALT and aspartate aminotransferase (AST) levels were measured using a Mindray BC-5000 automatic blood cell analyzer, a BS-600 automatic biochemical analyzer and a CL-2000i chemiluminescence immunoassay analyzer (all purchased from Mindray Bio-medical Electronics Co., Ltd.), respectively. The patients were divided into four subgroups based on age: i) $<1$ year old; ii) 1-3 years old; iii) 3-5 years old; and iv) $\geq 5$ years old.

Color Doppler echocardiography. Color Doppler echocardiography was carried out when the patient was initially hospitalized, as well as 2 and 5 weeks after IVIG administration. ' $Z_{\text {worst }}$ ' was defined as the higher $\mathrm{Z}$-scores of the right coronary artery and the left anterior descending coronary artery at any point during the active disease. The aortic root was measured parallel to the long axis near the sternum over the mid-systolic phase. A Z-score $\geq 2.0$ for the aortic sinus after normalizing to the body surface area was considered as dilation (10). According to The American Heart Association's adjusted standards, expansion of the right coronary artery and left anterior descending coronary artery is defined as $\mathrm{Z}_{\text {worst }} \geq 2.5$ (standard deviation of mean internal diameter standardized by body surface area) (8). The patients in the CAL group were divided into three grades based on the severity of dilation. The first grade involved small aneurysms or local dilation (mild) in 0-5 years old children whose coronary artery diameter was $<4 \mathrm{~mm}$, or in children $>5$ years old whose coronary artery diameter was 1.5 times less than that in the adjacent sectors. The second grade involved medium-sized aneurysms (moderate) in children aged 0-5 years whose coronary artery diameter was $4-8 \mathrm{~mm}$, or in children $>5$ years old whose coronary artery diameter in the measured segment was 1.5-4 times less than that in the nearby sections. The third grade included giant aneurysms (severe) in children aged $0-5$ years whose coronary artery diameter was $>8 \mathrm{~mm}$ or in children $>5$ years old whose artery segment's diameter was 4 times larger compared with the nearby sections (11). Cardiac echocardiography was conducted for follow-up in these children, 6 months, 1 year and 2 years after discharge.

Statistical analysis. Statistical analysis was conducted using STATA 14.0 software (StataCorp LP) and GraphPad Prism 7.0 (GraphPad Software, Inc.) was used to construct figures. The measurement data are presented as the mean \pm standard deviation, and the enumeration data are presented as cases (\%). For continuous variables analysis between CAL and NCAL groups, Mann-Whitney U test or t-test was utilized. Differences in the classified variables was compared using the Kruskal-Wallis rank-sum test, Fisher's exact test or $\chi^{2}$ test. Kendall's rank correlation was used to analyze the correlation between rank variables. The general data, blood routine parameters and biochemical indexes between the CAL and NCAL groups were included in the logistic regression analysis model. Their impacts on CAL occurrence were analyzed using the stepwise regression technique. The test level of variables included in the equation was 0.10 , and the retention level of variables was 0.015 . Multiple linear regression was performed between coronary artery diameter and blood routine, and biochemical parameters. $\mathrm{P}<0.05$ was considered to indicate a statistically significant difference.

\section{Results}

Patient characteristics. Among the total 102 cases, 47 cases $(46.08 \%)$ presented with CAL complications. This included 11 cases of right coronary artery dilatation, 16 cases of left coronary artery dilatation, 6 cases of giant coronary artery aneurysm and 14 cases of bilateral coronary artery dilatation (Table I). The ratio of male to female cases was 1.37:1 (59:43), and the average age was $2.5 \pm 1.9$ years old. No significant differences were identified in the occurrence of CAL among various age groups $(\mathrm{P}=0.176$; Table I). Although, there was a difference in the severity of CAL among different age groups $(\mathrm{P}=0.063)$ and a significant correlation was identified between them ( $\mathrm{P}=0.004$; Table II; Fig. 1).

Univariate analysis. In the CAL group, fever duration, levels of NT-proBNP, cTnI, IVIG resistance, CRP, ESR, platelet, ALT and AST were significantly higher compared with the NCAL group (all $\mathrm{P}<0.05$; Tables III and IV). The serum ALB levels were significantly lower in the CAL group compared with the NCAL group $(\mathrm{P}<0.05$; Tables III and IV).

Logistic regression analysis. Logistic regression analysis was conducted with the coronary artery lesions as depen- 
Table I. Occurrence rate of CAL in different age groups.

\begin{tabular}{|c|c|c|c|c|c|}
\hline Age group & $<1$ year, $\mathrm{n}=29$ & $1-3$ year(s), $n=41$ & $3-5$ years, $n=19$ & $\geq 5$ year, $n=13$ & Total, $\mathrm{n}=102$ \\
\hline Left coronary artery dilatation, $\mathrm{n}(\%)$ & $6(20.69)$ & $6(14.63)$ & $2(10.53)$ & $2(15.38)$ & $16(15.69)$ \\
\hline Right coronary artery dilatation, $\mathrm{n}(\%)$ & $4(13.79)$ & $4(9.76)$ & $2(10.53)$ & $1(7.69)$ & $11(10.78)$ \\
\hline Bilateral coronary artery dilation, $\mathrm{n}(\%)$ & $6(20.69)$ & $5(12.20)$ & $2(10.53)$ & $1(7.69)$ & $14(13.73)$ \\
\hline Giant coronary artery aneurysm, n (\%) & $2(6.90)$ & $3(7.32)$ & $1(5.26)$ & $0(0.00)$ & $6(5.88)$ \\
\hline Total, n (\%) & $18(62.07)$ & $18(43.90)$ & $7(36.84)$ & $4(30.77)$ & $47(46.08)$ \\
\hline
\end{tabular}

Incidence of CAL in different age groups was statistically analyzed, giving $\chi^{2}=4.941, \mathrm{P}=0.176$. CAL, coronary artery lesion.

Table II. Severity of CAL in different age groups.

\begin{tabular}{|c|c|c|c|c|c|}
\hline Age group & $<1$ year, $n=29$ & $1-3$ year $(s), n=41$ & $3-5$ years, $n=19$ & $\geq 5$ year, $n=13$ & Total, $n=102$ \\
\hline None, n (\%) & $11(37.93)$ & $23(56.10)$ & $12(63.16)$ & $9(69.23)$ & $55(53.92)$ \\
\hline Mild, n (\%) & $3(10.34)$ & $8(19.51)$ & $4(21.05)$ & $2(15.38)$ & $17(16.67)$ \\
\hline Moderate, n (\%) & $6(20.69)$ & $5(12.20)$ & $2(10.53)$ & $2(15.38)$ & $15(14.71)$ \\
\hline Severe, n (\%) & $9(31.03)$ & $5(12.20)$ & $1(5.26)$ & $0(0.00)$ & $15(14.71)$ \\
\hline
\end{tabular}

Severity of CAL in different age groups was statistically analyzed, giving $\chi^{2}=7.287, \mathrm{P}=0.063$. In the Kendall's $\tau \mathrm{b}$ correlation analysis, between the severity of CAL and the different ages, Kendall's $\tau b=-0.246, P=0.004$. CAL, coronary artery lesion.

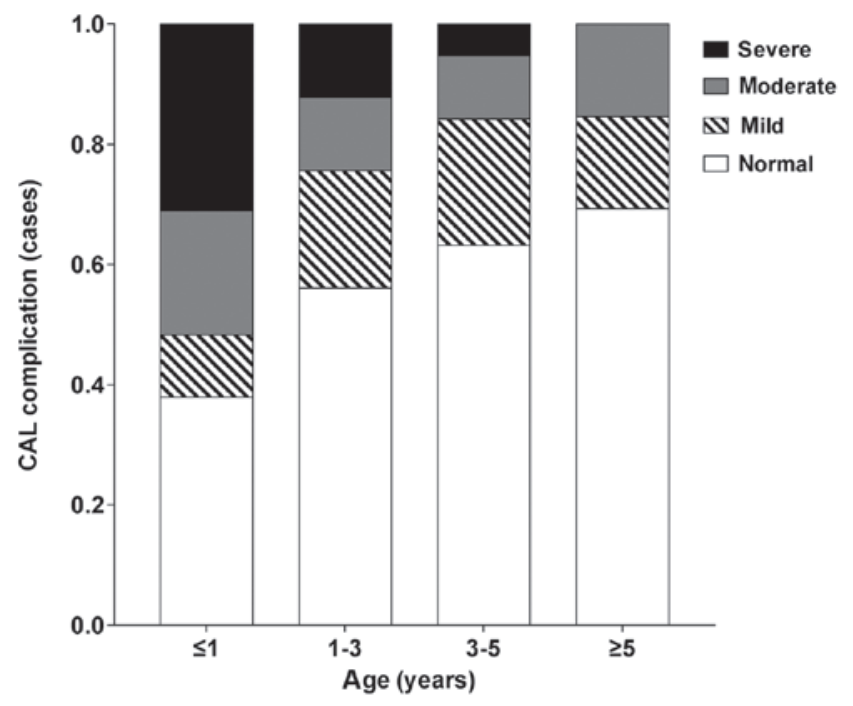

Figure 1. Severity of CAL in different age groups. CAL, coronary artery lesion.

dent variables and the indices with significant differences in univariate analysis as independent variables. The assignments of continuous variables in routine blood parameters and the biochemical indicators are presented in Table V. The logistic regression analysis results demonstrated that fever duration $(\mathrm{OR}=2.014)$, NT-proBNP (OR=3.004), cTnI level $(\mathrm{OR}=2.638)$, ESR (OR=1.461) and CRP elevation $(\mathrm{OR}=1.094)$ were the parameters affecting $\mathrm{CAL}$ in $\mathrm{KD}\left(\mathrm{R}^{2}=0.691\right.$; Table VI).

Logistic regression (stepwise regression method) was also conducted by taking the pre-treatment coronary artery diameter $(\mathrm{mm})$ and the indices with significant differences in univariate analysis as independent variables and with the coronary artery lesions as dependent variables. Finally, $\mathrm{Y}(\mathrm{CAL}=1 / \mathrm{NCAL}=0)=0.24 *$ coronary artery diameter $+0.59 *$ fever duration $+1.15 * \mathrm{NT}$-proBNP +0.83 $*$ cTnI + $0.61 *$ ESR-6.73 $\left(\mathrm{R}^{2}=0.952\right)$. This improved the determinant coefficient of the logistic regression model.

Significant differences were identified in dilated lesions within the patients with CAL at different levels in the follow-up $\left(\chi^{2}=11.48, \mathrm{P}=0.003\right)$. The follow-up of mild CAL patients was better than that of moderate and severe CAL patients $\left(\chi^{2}=6.48\right.$ and 11.48 , respectively, $\mathrm{P}<0.01)$. The diameter of coronary artery in the recovery period of patients with different severity in CAL group was significantly reduced. The t-values of mild, moderate and severe patients were 6.31, 6.03 and 5.00, respectively, P-values were <0.001 (Table VII; Fig. 2).

Multiple linear regression. The blood routine parameters and biochemical indexes of the patients were taken as independent variables and the coronary artery diameters after the treatment was taken as a dependent variable to establish a multiple linear regression model. The results showed the coronary artery diameter after treatment $=0.21 *$ fever duration +0.73 $*$ NT-proBNP $+0.94 * \mathrm{cTnI}+0.59 * \mathrm{ESR}+0.47 *$ C-reactive protein-1.58 $\left(\mathrm{R}^{2}=0.636\right)$.

\section{Discussion}

$\mathrm{KD}$ is an inflammatory febrile disease occurring in children and is the initial cause for childhood-acquired heart illness. Coronary artery aneurysm is a severe KD complication, which causes ischemic heart disease, myocardial infarction and unexpected cardiac death (1). Coronary artery Z-score measurement using echocardiography within the acute phase of KD not only provides vascular health information, but 
Table III. Basic information of the patients.

\begin{tabular}{lcccc}
\hline Characteristics & CAL group, $\mathrm{n}=47$ & NCAL group, $\mathrm{n}=55$ & $\chi^{2} / \mathrm{t}$ & P-value \\
\hline Age, year & $2.8 \pm 1.8$ & $2.3 \pm 2.0$ & 0.53 & 0.604 \\
Sex, male/female & $28 / 19$ & $31 / 24$ & 0.11 & 0.743 \\
Days of fever & $8.37 \pm 2.53$ & $6.05 \pm 1.82$ & 2.14 & 0.035 \\
Fever, $\mathrm{n}(\%)$ & $47(100)$ & $55(100)$ & - & - \\
Conjunctivitis, $\mathrm{n}(\%)$ & $40(85.11)$ & $47(85.45)$ & 0.00 & 0.961 \\
Stomatitis, $\mathrm{n}(\%)$ & $41(87.23)$ & $44(80.00)$ & 0.95 & 0.328 \\
Erythra, $\mathrm{n}(\%)$ & $32(68.09)$ & $37(67.27)$ & 0.01 & 0.930 \\
Lymphadenopathy, $\mathrm{n}(\%)$ & $27(57.45)$ & $31(56.36)$ & 0.01 & 0.912 \\
Changes in limbs, $\mathrm{n}(\%)$ & $5(10.64)$ & $6(10.91)$ & 0.00 & 0.965 \\
\hline
\end{tabular}

CAL, coronary artery lesion; NCAL, non-coronary artery lesion.

Table IV. Results of blood routine examination and biochemical factor of the patients.

\begin{tabular}{|c|c|c|c|c|}
\hline Parameter & CAL group, $n=47$ & NCAL group, $n=55$ & $\chi^{2 / t}$ & P-value \\
\hline WBC, $\times 10^{9} / 1$ & $18.14 \pm 6.36$ & $17.23 \pm 6.73$ & 0.31 & 0.762 \\
\hline PLT, $10^{3} / \mathrm{mm}^{3}$ & $643.65 \pm 104.37$ & $410.43 \pm 145.91$ & 3.20 & $<0.001$ \\
\hline $\mathrm{HGB}, \mathrm{g} / \mathrm{l}$ & $109.46 \pm 15.36$ & $113.73 \pm 17.59$ & 0.46 & 0.651 \\
\hline $\mathrm{ALB}, \mathrm{g} / \mathrm{l}$ & $19.63 \pm 5.91$ & $36.57 \pm 10.24$ & 5.30 & $<0.001$ \\
\hline IVIG resistance $(\%)$ & $29(61.70)$ & $17(30.91)$ & 9.71 & 0.002 \\
\hline $\mathrm{ESR}, \mathrm{mm} / \mathrm{h}$ & $70.46 \pm 23.25$ & $48.70 \pm 20.92$ & 2.04 & 0.045 \\
\hline CRP, mg/l & $76.21 \pm 25.33$ & $47.06 \pm 15.46$ & 2.72 & 0.008 \\
\hline ALT, IU/1 & $51.89 \pm 17.26$ & $33.70 \pm 18.24$ & 3.49 & 0.001 \\
\hline AST, IU/1 & $78.51 \pm 16.32$ & $39.48 \pm 15.47$ & 4.32 & $<0.001$ \\
\hline NT-proBNP, ng/ml & $1.78 \pm 2.85$ & $1.15 \pm 1.72$ & 3.86 & $<0.001$ \\
\hline $\mathrm{cTnI}, \mathrm{ng} / \mathrm{ml}$ & $0.02 \pm 0.04$ & $0.01 \pm 0.01$ & 2.10 & 0.039 \\
\hline
\end{tabular}

CAL, coronary artery lesion; NCAL, non-coronary artery lesion; WBC, white blood cell; PLT, platelets; HGB, hemoglobin; ALB, albumin; IVIG, intravenous immunoglobulin; ESR, erythrocyte sedimentation rate; CRP, C-reactive protein; ALT, alanine aminotransferase; AST, aspartate aminotransferase; NT-proBNP, N-terminal pro B-type natriuretic peptide; cTnI, cardiac troponin I.

also provides the risk assessment of cardiovascular disease and aids in clinical decision-making (12). CAL in patients with KD leads to atherosclerotic vascular remodeling during recovery by intimal hyperplasia and neovascularization after vasculitis (13). Based on the current recommendations, it is stated that echocardiography must be conducted during the diagnosis for complex KD cases without any obvious signs of coronary artery disease, then again at 1-2 weeks and 4-6 weeks following treatment. More frequent evaluation is required for patients with progressive abnormalities to detect thrombosis and aneurysms $(14,15)$.

The signs and symptoms, clinical data and echocardiographic results of 200 children with KD were analyzed in a previous study and it was identified that the signs and symptoms of KD in infants $<3$ months old were varied, and these patients were exposed to a higher risk of coronary artery anomalies; nevertheless, barring the patients suffering from giant coronary artery aneurysms, all other patients successfully recovered after an average follow-up period of 18 months with no complications (16). A previous retrospective study performed by Cameron et al (17) compared the severity of coronary artery dilatation in 93 infants within the age of 1 year and 170 children older than 1 year old treated at the same time. The previous study identified that there was a significantly higher average maximum Z-score in the infants than the older children. Furthermore, the infants had a higher occurrence of aneurysms compared with the older children (11 vs. 3\% for moderate aneurysms and 8 vs. $1 \%$ for giant aneurysms) (15). These previous studies also suggested that as the children's age increases, the occurrence of coronary artery dilatation and the severity of arterial dilatation were reduced.

The majority of patients with KD recover well after treatment; however, coronary artery aneurysms may develop in $\sim 25 \%$ of untreated cases (18). Early diagnosis and treatment together with follow-up by echocardiography play important roles for positive outcomes. The risk of cardiovascular complications can be considerably reduced by combining IVIG and aspirin over 10 days of KD onset (15). In total, 10-15\% of children with KD do not respond positively against initial IVIG and there is an elevated risk of CAL in these children (19). 
Table V. Assignment table of logistic regression analysis.

\begin{tabular}{|c|c|c|c|c|}
\hline \multirow[b]{2}{*}{ Parameter } & \multicolumn{3}{|c|}{ Assignment } & \multirow[b]{2}{*}{ Weight coefficient } \\
\hline & $<0$ & 1 & 2 & \\
\hline PLT, $x 10^{3} / \mathrm{mm}^{3}$ & $<400$ & $\geq 400,<600$ & $\geq 600$ & 1 \\
\hline ALB, $\mathrm{g} / 1$ & $<20$ & $\geq 20,<40$ & $\geq 40$ & 1 \\
\hline $\mathrm{ESR}, \mathrm{mm} / \mathrm{h}$ & $<50$ & $\geq 50,<70$ & $\geq 70$ & 1 \\
\hline CRP, mg/l & $<50$ & $\geq 50,<70$ & $\geq 70$ & 1 \\
\hline ALT, IU/1 & $<30$ & $\geq 30,<50$ & $\geq 50$ & 1 \\
\hline AST, IU/1 & $<40$ & $\geq 40,<70$ & $\geq 70$ & 1 \\
\hline NT-proBNP, ng/ml & $<1.1$ & $\geq 1.1,<1.8$ & $\geq 1.8$ & 1 \\
\hline cTnI, ng/ml & $<0.01$ & $\geq 0.01,<0.02$ & $\geq 0.02$ & 1 \\
\hline
\end{tabular}

PLT, platelets; ALB, albumin; ESR, erythrocyte sedimentation rate; CRP, C-reactive protein; ALT, alanine aminotransferase; AST, aspartate aminotransferase; NT-proBNP, N-terminal pro B-type natriuretic peptide; cTnI, cardiac troponin I.

Table VI. Result of logistic regression analysis.

\begin{tabular}{|c|c|c|c|c|c|}
\hline Parameter & $\beta$ & $\mathrm{SE}$ & $\chi^{2}$ (wald) & P-value & OR \\
\hline Constant & -25.04 & 11.35 & 4.87 & & \\
\hline Days of fever & 0.7 & 0.32 & 4.79 & 0.03 & 2.014 \\
\hline NT-proBNP & 1.1 & 0.34 & 10.47 & 0.00 & 3.004 \\
\hline cTnI & 0.97 & 0.44 & 4.86 & 0.03 & 2.638 \\
\hline ESR & 0.38 & 0.189 & 4.04 & 0.04 & 1.461 \\
\hline CRP & 0.09 & 0.04 & 5.06 & 0.02 & 1.094 \\
\hline
\end{tabular}

ESR, erythrocyte sedimentation rate; CRP, C-reactive protein; NT-proBNP, N-terminal pro B-type natriuretic peptide; cTnI, cardiac troponin I; $\mathrm{OR}$, odds ratio.

A previous meta-analysis performed by Baek and Song (19) on 2,745 patients from 12 studies demonstrated that raised NT-proBNP, ALT, bilirubin and CRP, and reduced ALB and sodium levels were risk factors for non-response to primary treatment with IVIG. A multicenter retrospective study carried out in 38 Korean hospitals identified that serum NT-proBNP levels and the percentage of polymorphonuclear leukocytes in the IVIG resistant group were considerably higher compared with the control group. Based on the correlation between NT-proBNP levels and IVIG resistance, it was suggested that NT-proBNP may be valuable in prediction prognosis (20).

In recent years, developments in molecular biology and clinical medicine techniques have made it possible to discover biomarkers as a powerful instrument for stratifying the risk and predicting the prognosis of cardiovascular diseases (21). Myocardial cell stress-related biomarkers are raised in most patients suffering from acute KD; NT-proBNP is associated with inflammation, while oxidative stress markers and echocardiographic results indicate diastolic dysfunction (22). Myocardial cells release NT-proBNP against the inflammatory cytokines and ventricular dilatation. It was primarily utilized in diagnosing cardiac disease in adults, and it was demonstrated that this marker was effective in diagnosing and treating patients with KD as well (23). In the early stage of KD, myocardial inflammation is common. NT-proBNP may be a more useful biomarker for diagnosing KD compared with the highly sensitive CRP (19). The mean NT-proBNP levels in patients with complete and incomplete KD was considerably higher than that of age-matched patients with simple fever (22). In addition, the mean NT-proBNP levels in patients with complete KD was higher than that of patients with incomplete $\operatorname{KD}(23,24)$. A previous study identified that high NT-proBNP levels exist in patients with KD who are resistant to IVIG treatment as well as those who have a coronary artery aneurysm, indicating its prognostic utility (24). There is a high risk of incomplete $\mathrm{KD}$ and early CAL in infants $<3$ months compared with older patients. Therefore, it is recommended that infants with fever lasting for $>2$ days are evaluated by echocardiography and also for NT-proBNP levels, irrespective of the presence or absence of other KD-related symptoms (25). The present results suggested that NT-proBNP levels in the CAL group were considerably higher when compared with the NCAL group. Based on the logistic regression analysis, it was observed that the OR of NT-proBNP was 3.004, indicating that there is an association between NT-proBNP levels and the incidence of CAL.

cTnI is a cardiomyocyte injury or death marker and is increased in both patients with KD and fever controls (24). Based on unexpected increments in cTnI in the majority of 
Table VII. Results of cardiac ultrasound examination in the coronary artery lesion group in the follow-up.

Severity of disease

Result

Mild, $\mathrm{n}=17$

Moderate, $\mathrm{n}=15$

Severe, $\mathrm{n}=15$

Dilated lesions $^{\mathrm{a}}$

Return to normal

17

0

0

Continued expansion

Coronary artery diameter, $\mathrm{mm}$

Before treatment

After treatment
$3.89 \pm 0.56$
$2.80 \pm 0.44$

$4.51 \pm 0.72$

$3.08 \pm 0.57^{\mathrm{b}}$
$9.78 \pm 1.85$

7

5

3

$6.15 \pm 2.12^{\mathrm{b}}$

aThere was a significant difference in dilated lesions within the patients with CAL at different levels at the follow-ups. The follow-up of mild CAL patients was better than that of moderate and severe CAL patients (all $\mathrm{P}<0.01$ ). CAL, coronary artery lesion. ${ }^{\mathrm{b}} \mathrm{Compared}$ with before treatment, the difference in the diameter of coronary artery was statistically significant, $\mathrm{P}<0.001$.

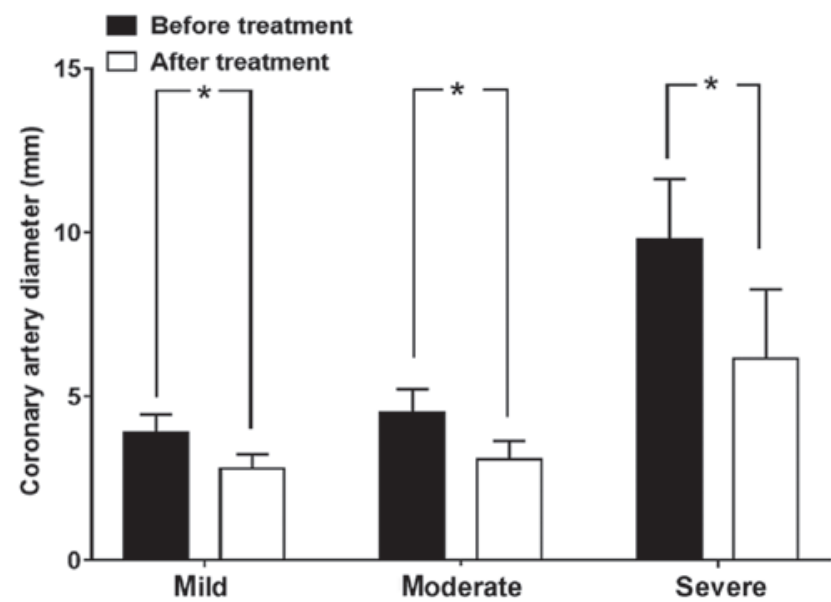

Figure 2. Comparison of coronary artery diameter before and after treatment in the coronary artery lesion group. ${ }^{*} \mathrm{P}<0.05$.

patients with KD in convalescence, it was suggested that myocardial cell damage occurs even after the systemic inflammatory markers reduced to normal levels. In all patients tested 1-2 years after disease onset, cTnI levels returned to normal, further suggesting that the previous increase in cTnI was related to the myocardial cell damage or death (26). In patients with incomplete $\mathrm{KD}$, the acute and sub-acute age-corrected hemoglobin levels were reduced; however, the sub-acute platelet counts increased in patients with complete KD. Furthermore, it is known that the platelet counts in KD patients are positively correlated with fever duration, and a longer inflammation period may be reflected in the increase in platelet counts (27). Jeon et al (28) observed that coronary artery aneurysms developed in 30 of 392 patients with KD within a month of diagnosis while in $20 \%$ they persisted even after 1 year of follow-up. High platelet counts and long-term fever are independent indicators of coronary artery aneurysm immediately after diagnosis. According to the univariate analysis in a previous study, increased white blood cell count and the primary coronary artery severity were independent risk factors for the persistence of enhanced coronary artery aneurysms. Nevertheless, no considerable difference was identified in the multivariate analysis (15). Previous studies have demonstrated that platelet counts and age-corrected hemoglobin can be utilized not only as specific indicators of KD but also as predictors of coronary artery dilatation in patients with complete and incomplete KD, respectively. Elevated platelet counts and CRP levels, and lower hemoglobin and ALB levels are also risk factors for acute coronary artery aneurysms $(12,29)$. In general, these previous studies were in agreement with the univariate analysis in the present study. However, platelet count, hemoglobin and ALB were not considered for logistic regression in the present study as independent factors for the incidence of CAL.

The clinical markers analyzed in the present study are very simple to measure, and most are associated with significant differences in patients with CAL and NCAL. However, the present study had some limitations. First, there were numerous indicators for analysis and classifying the patients based on the age was too narrow. Consequently, future studies should aim to increase the sample size and carry out a multi-center study to validate the results obtained in the present study. There was a correlation between clinical markers and KD with concurrent CAL; however, more data are required to validate these markers as risk factors for disease incidence. Ultimately, the markers evaluated in the logistic regression analysis had shown differences in the univariate analysis; therefore, the key risk factors may be eliminated due to the confounding factors.

In conclusion, color Doppler echocardiography is able to measure the degree of illness in patients with KD and predict the outcome of coronary artery disease in real time since it is useful for both early diagnosis and long-term follow-up. Fever duration, NT-proBNP, cTnI levels, ESR and elevated CRP may be high-risk factors for CAL in KD. The fever duration, cTnI level, NT-proBNP and ESR were also correlated with coronary artery diameter. Comprehensive use of these clinical parameters may more accurately determine the occurrence of CAL in KD.

\section{Acknowledgements}

Not applicable. 


\section{Funding}

The present study was supported by The Natural Science Foundation of Inner Mongolia (grant no. 2018MS08080).

\section{Availability of data and materials}

The datasets used and/or analysed during the current study are available from the corresponding author on reasonable request.

\section{Authors' contributions}

MX helped collect the data, perform the ultrasound diagnosis and follow-up, write the manuscript and perform the statistical analysis. JW contributed in the conception and design of the study, data gathering, ultrasound diagnosis and follow-up, and manuscript guidance. All authors read and approved the final manuscript.

\section{Ethics approval and consent to participate}

This prospective work was approved by The Ethics Committee of the Affiliated Hospital of Inner Mongolia Medical University, and informed consents were obtained from the legal guardians of the enrolled children.

\section{Patient consent for publication}

Not applicable.

\section{Competing interests}

The authors declare that they have no competing interests.

\section{References}

1. Singh S, Vignesh P and Burgner D: The epidemiology of Kawasaki disease: A global update. Arch Dis Child 100: 1084-1088, 2015.

2. Hedrich CM, Schnabel A and Hospach T: Kawasaki Disease. Front Pediatr 6: 198, 2018.

3. JCS Joint Working Group: Guidelines for diagnosis and management of cardiovascular sequelae in Kawasaki disease (JCS 2013). Digest version. Circ J 78: 2521-2562, 2014.

4. McCrindle BW and Cifra B: The role of echocardiography in Kawasaki disease. Int J Rheum Dis 21: 50-55, 2018.

5. Argyropoulou OD, Protogerou AD and Sfikakis PP: Accelerated atheromatosis and arteriosclerosis in primary systemic vasculitides: Current evidence and future perspectives. Curr Opin Rheumatol 30: 36-43, 2018.

6. Kato M, Ayusawa M, Watanabe H, Komori A, Abe Y, Nakamura T, Kamiyama $\mathrm{H}$ and Takahashi S: Cardiac function on 3-D speckle tracking imaging and cytokines in Kawasaki disease. Pediatr Int (Roma) 60: 342-348, 2018.

7. Singh S, Jindal AK and Pilania RK: Diagnosis of Kawasaki disease. Int J Rheum Dis 21: 36-44, 2018.

8. Pilania RK, Bhattarai D and Singh S: Controversies in diagnosis and management of Kawasaki disease. World J Clin Pediatr 7: 27-35, 2018.

9. Newburger JW, Takahashi M, Gerber MA, Gewitz MH, Tani LY, Burns JC, Shulman ST, Bolger AF, Ferrieri P, Baltimore RS, et al; Committee on Rheumatic Fever, Endocarditis, and Kawasaki Disease, Council on Cardiovascular Disease in the Young, American Heart Association: Diagnosis, treatment, and long-term management of Kawasaki disease: A statement for health professionals from the Committee on Rheumatic Fever, Endocarditis, and Kawasaki Disease, Council on Cardiovascular Disease in the Young, American Heart Association. Pediatrics 114: 1708-1733, 2004.
10. Ravekes WJ, Colan SD, Gauvreau K, Baker AL, Sundel RP, van der Velde ME, Fulton DR and Newburger JW: Aortic root dilation in Kawasaki disease. Am J Cardiol 87: 919-922, 2001.

11. Noto N, Komori A, Ayusawa M and Takahashi S: Recent updates on echocardiography and ultrasound for Kawasaki disease: Beyond the coronary artery. Cardiovasc Diagn Ther 8: 80-89, 2018.

12. Ha KS, Jang GY, Lee J, Lee KC and Son CS: Laboratory Markers in Incomplete Kawasaki Disease according to Coronary Artery Outcome. Korean Circ J 48: 287-295, 2018.

13. Cameron SA, Robinson JD, Carr MR and Patel A: Giant coronary artery aneurysms in an infant with Kawasaki disease: Evaluation by echocardiography and computed tomographic angiography. Echocardiography 35: 1692-1694, 2018.

14. de Ferranti SD, Gauvreau K, Friedman KG, Tang A, Baker AL, Fulton DR, Tremoulet AH, Burns JC and Newburger JW: Association of Initially Normal Coronary Arteries With Normal Findings on Follow-up Echocardiography in Patients With Kawasaki Disease. JAMA Pediatr 172: e183310, 2018.

15. Dionne A and Dahdah N: Myocarditis and Kawasaki disease. Int J Rheum Dis 21: 45-49, 2018.

16. Li W, Zhang L, Huang P and Zhang Z: Clinical features and mid-term follow-up in infants younger than 3 months with Kawasaki disease in a Chinese population. J Paediatr Child Health 55: 523-527, 2019.

17. Cameron SA, Carr M, Pahl E, DeMarais N, Shulman ST and Rowley AH: Coronary artery aneurysms are more severe in infants than in older children with Kawasaki disease. Arch Dis Child 104: 451-455, 2019.

18. Toyono M, Takagi D, Oyamada J, Shimada S, Aoki-Okazaki M and Takahashi T: Coronary artery aneurysm after kawasaki disease in a single coronary artery. Circ J 77: 2409-2411, 2013.

19. Baek JY and Song MS: Meta-analysis of factors predicting resistance to intravenous immunoglobulin treatment in patients with Kawasaki disease. Korean J Pediatr 59: 80-90, 2016.

20. Kim MK, Song MS and Kim GB: Factors Predicting Resistance to Intravenous Immunoglobulin Treatment and Coronary Artery Lesion in Patients with Kawasaki Disease: Analysis of the Korean Nationwide Multicenter Survey from 2012 to 2014. Korean Circ J 48: 71-79, 2018.

21. Maggio MC, Corsello G, Prinzi E and Cimaz R: Kawasaki disease in Sicily: Clinical description and markers of disease severity. Ital J Pediatr 42: 92, 2016.

22. Lee SH, Song ES, Yoon S, Hong S, Cho HJ, Yang EM, Eom GH, Kang G and Cho YK: Usefulness of Age-Stratified N-Terminal Prohormone of Brain Natriuretic Peptide for Diagnosing Kawasaki Disease. Dis Markers 2017: 6263121, 2017.

23. Dionne A and Dahdah N: A Decade of NT-proBNP in Acute Kawasaki Disease, from Physiological Response to Clinical Relevance. Children (Basel) 5: E141, 2018.

24. Sato YZ, Molkara DP, Daniels LB, Tremoulet AH, Shimizu C, Kanegaye JT, Best BM, Snider JV, Frazer JR, Maisel A, et al: Cardiovascular biomarkers in acute Kawasaki disease. Int $\mathrm{J}$ Cardiol 164: 58-63, 2013.

25. Satoh K, Wakejima Y, Gau M, Kiguchi T, Matsuda N, Takasawa R, Takasawa K, Nishioka M and Shimohira M: Risk of coronary artery lesions in young infants with Kawasaki disease: Need for a new diagnostic method. Int J Rheum Dis 21: 746-754, 2018.

26. Liu W, Liu C, Zhang L, Xie X, Gu X, Sang C, Xu M, Xu W and Jia H: Molecular basis of coronary artery dilation and aneurysms in patients with Kawasaki disease based on differential protein expression. Mol Med Rep 17: 2402-2414, 2018.

27. Chen J, Liu Y, Liu W and Wu Z: A meta-analysis of the biomarkers associated with coronary artery lesions secondary to Kawasaki disease in Chinese children. J Huazhong Univ Sci Technolog Med Sci 31: 705-711, 2011.

28. Jeon SK, Kim G, Ko H, Byun JH and Lee HD: Risk factors for the Occurrence and Persistence of Coronary Aneurysm in Kawasaki Disease. Korean J Pediatr 62: 138-143, 2019.

29. Kim HJ, Choi EH, Lim YJ and Kil HR: The Usefulness of Platelet-derived Microparticle as Biomarker of Antiplatelet Therapy in Kawasaki Disease. J Korean Med Sci 32: 1147-1153, 2017. 